\title{
The Influence of Emotionally Linked Odors on Autonomic Nerve Activities
}

\author{
Yasuto Nakanishi ${ }^{1,}$, , Yoshiaki Sato ${ }^{1}$, Hideomi Nakata ${ }^{1}$, Hidehiro Nakahara ${ }^{2}$, Yosimitsu Inoue ${ }^{3}$, \\ Hiroshi Kinoshita ${ }^{4}$
}

${ }^{1}$ Department of Sports and Health Science, Faculty of Sports and Health Science, Osaka Sangyo University, Osaka, Japan

${ }^{2}$ Department of Acupuncture, Faculty of Health Science, Morinomiya University of Medical Sciences, Osaka, Japan

${ }^{3}$ Department of Health Science, Faculty of Human Science, Osaka International University, Osaka, Japan

${ }^{4}$ Department of Health and Nutrition, Science Faculty of Health Science, Osaka-Aoyama University, Osaka, Japan

Email address:

nakanishi@spo.osaka-sandai.ac.jp (Y. Nakanishi), yassy0914@hotmail.co.jp (Y. Nakanishi)

${ }^{*}$ Corresponding author

\section{To cite this article:}

Yasuto Nakanishi, Yoshiaki Sato, Hideomi Nakata, Hidehiro Nakahara, Yosimitsu Inoue, Hiroshi Kinoshita. The Influence of Emotionally Linked Odors on Autonomic Nerve Activities. American Journal of BioScience. Vol. 9, No. 4, 2021, pp. 147-152.

doi: 10.11648/j.ajbio.20210904.16

Received: July 18, 2021; Accepted: August 2, 2021; Published: August 11, 2021

\begin{abstract}
The limbic system, supporting emotions, behavioral motivation, and memory, in the brain receives coming odor before the sensory cortex. Therefore, certain odor can affect autonomic nerve system and physiological parameters due to smell-induced activity in the limbic system. The purpose of this study focused was to investigate the influence of odor, taking the level of arousal and valence into account, on autonomic nerve activities and physiological parameters. Eight healthy young males (20.6 $\pm 0.5 \mathrm{yr})$, who gave a response of high arousal $(6.8 \pm 0.5)$ and negative valence $(1.5 \pm 0.8)$ to the odor of vinegar (VNG), and an opposite response (arousal $2.3 \pm 0.8$, valence $6.6 \pm 0.4$ ) to the odor of orange (ORG), were selected as subjects. Heart rate and its variability (HRV), and blood pressure (BP) were evaluated for the subjects who were quietly seated, wore a plastic mask with a vapored odor-injection pipe, and breathed naturally the two aromas (VNG, and ORG). A no aroma condition was also included in the test as a control. Repeated measures ANOVA with a Turkey post-hoc test revealed a significant odor effect in the variables of HRV, and BP. VNG led to a higher ratio of low frequency /high frequency (LF/HF), and lower HF compared to the control ( $p<0.05, p<0.05$, respectively). VNG also had significantly higher diastolic and mean BP than ORG ( $<<0.05, p<0.05$, respectively). ORG, on the other hand, failed to show any significant odor effect on HRV and $\mathrm{BP}$ variables. $\mathrm{LF} / \mathrm{HF}$ can be a sympathetic nerve activity indicator while $\mathrm{HF}$ is known as a para-sympathetic nerve activity indicator. Our results of breathing VNG thus suggests that odors causing negative valence and high arousal can facilitate sympathetic nerve activity, and/or withdraw para-sympathetic nerve activity. A higher BP with VNG compared to ORG can also be attributed to the effect of these autonomic nerve activities. Consider all the various factors together, it can be concluded that some emotionally linked odors were strong enough to modulate autonomic nerve activities as well as the level of $\mathrm{BP}$ in resting individuals.
\end{abstract}

Keywords: Autonomic Nerve Activities, Arousal, Valence, HRV

\section{Introduction}

\subsection{Background of the Study}

The limbic system, supporting emotions, behavioral motivation, and memory, in the brain receives coming odor before the sensory cortex. Therefore, certain odor can affect autonomic nerve system and physiological parameters due to smell-induced activity in the limbic system. According to the study of Nagai [1], which dealt with rats, reported that sympathetic nerve activity was facilitated, and parasympathetic activity was suppressed by citrus odor. The studies of Nagai [1] and Nagai et al. [2] also reported that lavender odor caused opposite effects on parasympathetic activity. 
As for human studies, Haze et al. [3], reported that fragrance inhalation of patchouli and rose oils caused decrease in relative sympathetic activity, while Kanzaki [4] reported rose like odor caused increase in overall autonomic nerve activities. Yoshida and Saeki [5] reported that rosemary transiently increase sympathetic nerve activity, while Kawai [6] reported cineole, which is included in herbal essential oil such as rosemary and eucalyptus, did not bring about any changes in autonomic nerve activities. Thus, many studies have been pursued to evaluate the effects of odor on autonomic nerve activities, however, the results are not always consistent due probably to differences in stimulation methods and stimulation time among the studies. Therefore, systematic studies are thought to be needed to investigate the effects of odor on autonomic nerve activities.

Arousal and valence are proposed to represent fundamental dimensions of emotion. So, arousal and valence are other key issues to evaluate odor effects on autonomic nerve activities. Mori et al. [7], who studied effects of essential oil fragrances and preference on blood pressure (BP) and heart rate (HR) in healthy human, reported that the subjects with preference to lavender fragrance showed decrease in diastolic BP while the subjects with negative feeling to it showed increase in diastolic BP. They also reported that the subjects did not show any changes in BP and HR regardless of their preference to rosemary. Anderson et al. [8] reported that amygdala in the limbic system may represent pleasant and unpleasantness although intensity (used as a surrogate to arousal) and valence are often strongly associated in phenomenal experience such as odor, sound, and visual image. Wintson [9] et al. reported that the amygdala responds differentially to high (vs low) intensity odor for pleasant and unpleasant smells but not for neutral smell. They also mentioned that the amygdala codes neither arousal nor valence per se, but a combination reflects the overall value of a stimulus. So, we think that it is needed to consider the combination of valence and arousal when we investigate effects of odor on autonomic nerve activities. Zamadzki et al. [10] reported that emotions with high arousal and/or negative valence are associated with increase in BP, whereas emotions with low arousal and/or positive valence might be associated with dampening of BP.

Based on previous studies, young healthy males, who gave a response of high arousal and negative valence to the odor of vinegar (VNG) and an opposite response to the odor of orange (ORG), were selected as subjects in the present study. The present study was designed to systematically investigate the influence of odor, taking the level of arousal and valence into account, on autonomic nerve activities and physiological parameters such as HR, heart rate variability (HRV), BP, and salivary amylase.

\subsection{Research Hypotheses}

1) VNG, which produces negative valence and high arousal effects, enhances sympathetic activity and lowers parasympathetic activity.

2) ORG, which produces positive valence and low arousal effects, facilitates parasympathetic activity and lowers sympathetic activity.

\section{Methods}

\subsection{Subjects}

Initially, forty healthy young males $(20.6 \pm 0.5 \mathrm{yr})$ participated in the present study and eight healthy young males (20.1 $\pm 0.8 \mathrm{yr})$ among them were selected as subjects after the odor screening test. Before participating, the subjects were informed of all experimental procedures, the possible risks, and their voluntary withdrawal from the procedures at any time. Formal consent was obtained, all procedures conformed to the Declaration of Helsinki, and the protocol approved by the Ethical Committee of Osaka Sangyo University.

\subsection{Methods and Procedures}

\subsubsection{Odor Screening Tests}

There were seven sets of odor stimulation device, each of which consisted of a plastic mask with a vapored odor-injection pipe, and an aroma diffuser connected to the pipe. Each set of the device was used only for one odor.

The subjects quietly seated for five minutes, wore a plastic mask with a vapored odor-injection pipe, and breathed naturally an odor for ten seconds. Then, the subjects answered subjective survey, which has seven scales, to a diffused odor to assess arousal and valence to it (Figures 1 and 2). They repeated this odor screening test for seven times with an interval of two minutes to answer about all the seven odors (vinegar, peppermint, eucalyptus, grapefruits, orange, myrrh, and rosemary). The order of the odor screening tests was randomly assigned. Subjects avoided strenuous exercise and alcohol intake 24 hours prior to undertaking the screening tests and did not consume any food and caffeine for at least 3 hours prior to the test. We have carried out the screening test to select subjects who gave a response of high arousal and negative valence to one odor, and an opposite response to the other for the study. In addition, it was needed to obtain as many subjects as possible. So, all the results of the screening test were investigated to find out subjects who could fulfil the requirements mentioned above.

As the results, eight healthy young males, who gave a response of high arousal $(6.8 \pm 0.5)$ and negative valence $(1.5 \pm 0.8)$ to $\mathrm{VNG}$, and an opposite response (arousal $2.3 \pm 0.8$, valence $6.6 \pm$ 0.4 ) to ORG, were selected as subjects by the screening tests.

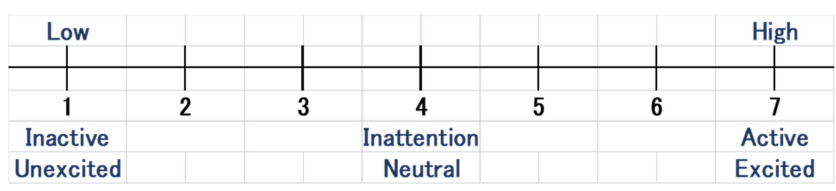

Figure 1. The scale of arousal.

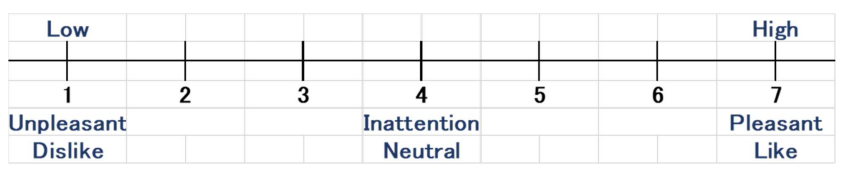

Figure 2. The scale of valence. 


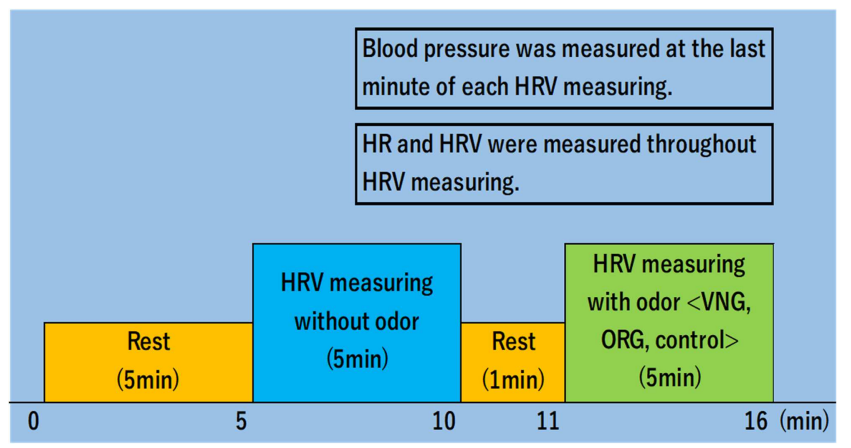

Figure 3. The schematic diagram of the present study.

\subsubsection{Autonomic Nerve Activity Measurement}

Autonomic nerve activities were estimated by HRV. The subjects entered the laboratory at least twenty minutes prior to the measurement to acclimatize the environment. The subject sat quietly for five minutes and HRV was measured for five minutes. After the measurement, the subjects rest for one minute and HRV was measured again with odor (VNG, ORG) for five minutes. A no aroma condition was also included in the measurement as a control. The schematic diagram of the present study is shown in Figure 3. HR and HRV were measured by a pulse analyzer (TAS9VIEW, YKC Corporation, Tokyo, Japan) during autonomic nerve activity measurement. BP was measured before and the last thirty seconds of the measurement by an automatic BP manometer (HEM-6311, Omuron Corporation, Kyoto, Japan). Salivary amylase was evaluated by a salivary amylase monitor (CM-21, Nipro Corporation, Osaka, Japan) before and after autonomic nerve activity measurement for the subjects who were quietly seated. The odors were given to the subjects by the same way as the screening tests.

Formal consent has been obtained by all subjects. All procedures have been conformed to the Declaration of Helsinki, and the protocol approved by the Ethical Committee of Osaka Aoyama University. Subjects avoided strenuous exercise and consumption of alcoholic beverages for at least 24 hours and did not consume any food or caffeine for at least 3 hours prior to undertaking each test procedure. Room temperature and humidity were kept constant throughout the testing at $25 \pm 1^{\circ} \mathrm{C}$ and $50 \pm 5 \%$, respectively.

\subsubsection{Data Analysis}

To examine the hypotheses, one-way repeated measures ANOVA, followed by a Turkey post-hoc test was performed on each of the parameters of autonomic nerve activity estimated from HR, HRV, BP, and salivary amylase measures. For all statistical measures, significance was established at the 0.05 level of probability.

Table 1. The effects of odors on autonomic nerve activities and blood pressure.

\begin{tabular}{|c|c|c|c|c|}
\hline \multirow{2}{*}{ Variables } & & \multicolumn{3}{|l|}{ Odors } \\
\hline & & Control & Orange & Vinegar \\
\hline \multirow{3}{*}{ HR } & bef. inhale (bpm) & $76.5 \pm 10.2$ & $73.4 \pm 11.6$ & $73.0 \pm 12.8$ \\
\hline & after inhale (bpm) & $75.5 \pm 11.0$ & $72.8 \pm 11.9$ & $75.1 \pm 13.8$ \\
\hline & $\Delta(\mathrm{bpm})$ & $-1.0 \pm 1.4$ & $-0.6 \pm 1.1$ & $2.1 \pm 2.6$ \\
\hline \multirow{3}{*}{$\mathrm{LF} / \mathrm{HF}$ ratio } & bef. inhale & $2.2 \pm 1.4$ & $1.4 \pm 1.2$ & $0.9 \pm 0.5$ \\
\hline & after inhale & $2.0 \pm 2.0$ & $1.9 \pm 2.1$ & $1.7 \pm 0.9$ \\
\hline & $\Delta$ & $-0.2 \pm 1.5$ & $0.5 \pm 1.1$ & $0.8 \pm 0.8$ \\
\hline \multirow{3}{*}{ HF norm } & bef. inhale & $35.9 \pm 11.5$ & $48.5 \pm 17.2$ & $54.6 \pm 12.1$ \\
\hline & after inhale & $43.7 \pm 19.6$ & $42.2 \pm 15.2$ & $39.9 \pm 14.6$ \\
\hline & $\Delta$ & $7.8 \pm 12.0 * *$ & $-6.3 \pm 11.8$ & $-14.7 \pm 12.4 * *$ \\
\hline \multirow{3}{*}{ SBP } & bef. Inhale (mmHg) & $125.1 \pm 13.9$ & $131.1 \pm 9.8$ & $126.1 \pm 11.1$ \\
\hline & after inhale (mmHg) & $133.0 \pm 15.2$ & $134.1 \pm 16.6$ & $136.8 \pm 15.9$ \\
\hline & $\Delta(\mathrm{mmHg})$ & $7.9 \pm 5.4$ & $3.0 \pm 9.3$ & $10.2 \pm 7.5$ \\
\hline \multirow{3}{*}{ DBP } & bef. Inhale (mmHg) & $76.0 \pm 9.3$ & $77.1 \pm 10.6$ & $77.6 \pm 13.4$ \\
\hline & after inhale (mmHg) & $79.1 \pm 11.7$ & $76.9 \pm 10.7$ & $86.4 \pm 12.7$ \\
\hline & $\Delta(\mathrm{mmHg})$ & $3.1 \pm 4.5$ & $-0.2 \pm 6.8^{*}$ & $8.8 \pm 6.5^{*}$ \\
\hline \multirow{3}{*}{ MBP } & bef. Inhale (mmHg) & $92.4 \pm 9.9$ & $95.1 \pm 8.9$ & $94.0 \pm 12.0$ \\
\hline & after inhale (mmHg) & $97.1 \pm 12.5$ & $96.0 \pm 10.1$ & $103.2 \pm 13.1$ \\
\hline & $\Delta(\mathrm{mmHg})$ & $4.7 \pm 3.8$ & $0.9 \pm 5.3^{*}$ & $9.2 \pm 6.6^{*}$ \\
\hline \multirow{4}{*}{ Salivary amylase } & bef. inhale (KIU/L) & $19.3 \pm 16.4$ & $18.3 \pm 14.5$ & $16.4 \pm 2.6$ \\
\hline & after inhale (KIU/L) & $19.5 \pm 16.5$ & $24.9 \pm 17.3$ & $21.1 \pm 5.4$ \\
\hline & $\Delta(\mathrm{KIU} / \mathrm{L})$ & $0.3 \pm 10.1$ & $6.6 \pm 11.7$ & $9.2 \pm 3.6$ \\
\hline & \multicolumn{3}{|c|}{$\mathrm{n}=8, * \mathrm{p}<0.05, * * \mathrm{p}<0.01$} & \\
\hline
\end{tabular}

CONTROL: without odor inhalation, ORG: orange odor inhalation, VNG: vinegar odor inhalation.

HF change for VNG were significantly higher than that for the control $(22.5, \mathrm{p}<0.01)$. DBP change for VNG was significantly higher than that for ORG $(9.1$ $\mathrm{mmHg}, \mathrm{p}<0.05)$ and MBP change for VNG was significantly higher than that for ORG $(8.3 \mathrm{mmHg}, \mathrm{p}<0.05)$. 


\section{Results}

The ANOVA showed that there was a main effect of odor in $\mathrm{LF} / \mathrm{HF}$ change ratio $[\mathrm{F}(2,14)=4.35, \mathrm{p}<0.05]$. Turkey post-hoc revealed that $\mathrm{LF} / \mathrm{HF}$ change ratio for VNG was 7.7 fold higher than that for the control $(p<0.05)$. The change ratio $=($ post-value - pre-value $) /$ pre-value $* 100$. The ANOVA also showed that there was a main effect of odor in HF change $[F(2,14)=7.12, p<0.01]$ and $H F$ change ratio $[F(2,14)=5.96, p<0.01]$. Turkey post-hoc revealed that $\mathrm{HF}$ change and HF change ratio for VNG were significantly higher $(22.6$ and $46.3 \%)$ than those for the control $(\mathrm{p}<0.01$, $\mathrm{p}<0.01$, respectively).

There was a main effect of odor in diastolic BP change $[\mathrm{F}(2,14)=4.52, \mathrm{p}<0.05]$ and its change ratio $[\mathrm{F}(2,14)=$ $4.36, \mathrm{p}<0.05]$. Turkey post-hoc revealed that diastolic BP change and its change ratio for VNG were significantly higher $(9.1 \mathrm{mmHg}$ and $11.9 \%)$ than those for $\mathrm{ORG}(\mathrm{p}<0.05$, $\mathrm{p}<0.05$, respectively). The ANOVA further showed that there was a main effect of odor in mean BP change $[\mathrm{F}(2,14)$ $=4.88, \mathrm{p}<0.01]$ and its change ratio $[\mathrm{F}(2,14)=4.83$, $\mathrm{p}<0.05]$. Turkey post-hoc revealed that mean BP change and its change ratio for VNG was significantly higher $(8.3$ $\mathrm{mmHg}$ and $9.1 \%)$ than those for ORG $(\mathrm{p}<0.05, \mathrm{p}<0.05$, respectively).

Contrary to our expectation, the odor effects on salivary amylase and HR did not reach the level of significance. All the results are shown in Table 1.

\section{Discussion}

The increase in LF/HF ratio component for vinegar was significantly higher than that for the control (Figure 4) and the decrease ratio in HF component for the vinegar was also significantly larger than that for the control (Figure 5).

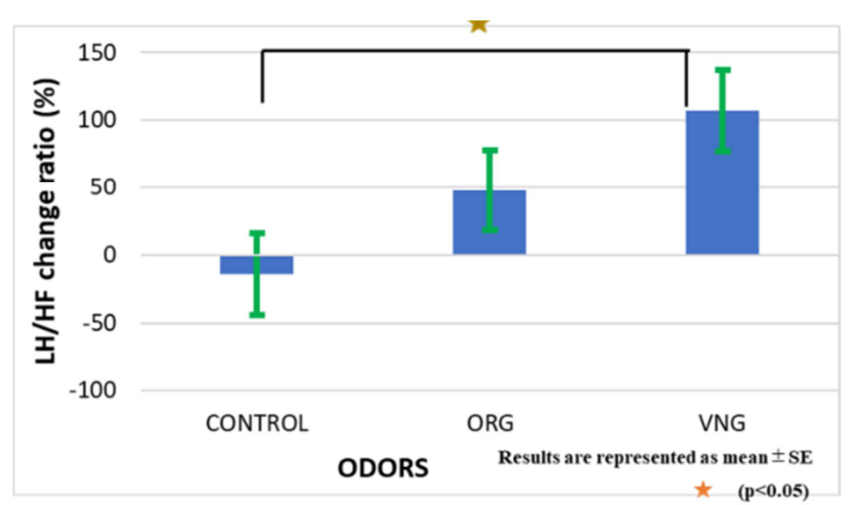

Figure 4. The effects of odors on sympathetic activities (LH/HF change ratio).

The change ratio $=($ post-value - pre-value $) /$ pre-value $* 100$. CONTROL: without odor inhalation, ORG: orange odor inhalation, VNG: vinegar odor inhalation. $\mathrm{LF} / \mathrm{HF}$ is known as a sympathetic nerve activity indicator. There was a main effect of odor in LF/HF change ratio $(\mathrm{p}<0.05)$. Turkey post-hoc revealed that $\mathrm{LF} / \mathrm{HF}$ change ratio for $\mathrm{VNG}$ was higher than that for the control $(\mathrm{p}<0.05)$.

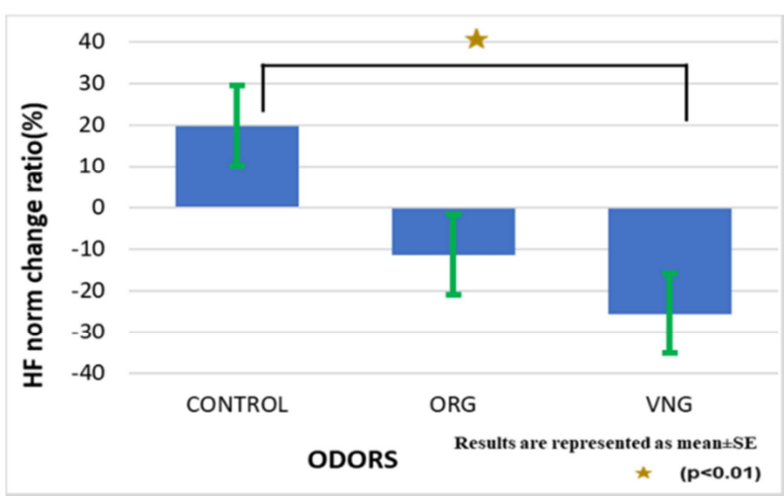

Figure 5. The effects of odors on para-sympathetic activities (HF norm change ratio).

The change ratio $=($ post-value - pre-value $) /$ pre-value $* 100$. CONTROL: without odor inhalation, ORG: orange odor inhalation, VNG: vinegar odor inhalation. HF is known as a para-sympathetic nerve activity indicator. There was a main effect of odor in HF change ratio $(p<0.05)$ and Turkey post-hoc revealed that $\mathrm{HF}$ change and $\mathrm{HF}$ change ratio for VNG were significantly higher than that for the control $(\mathrm{p}<0.01)$.

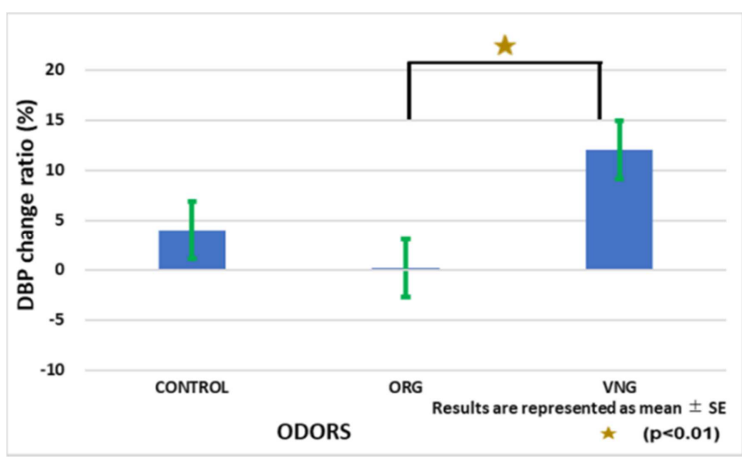

Figure 6. The effects of odors on DBP change ratio.

The change ratio $=($ post-value - pre-value $) /$ pre-value $* 100$. CONTROL: without odor inhalation, ORG: orange odor inhalation, VNG: vinegar odor inhalation. There was a main effect of odor in diastolic blood pressure change ratio $(p<0.05)$. Turkey post-hoc revealed that diastolic blood pressure change ratio for VNG were significantly higher than that for ORG $(\mathrm{p}<0.05)$.

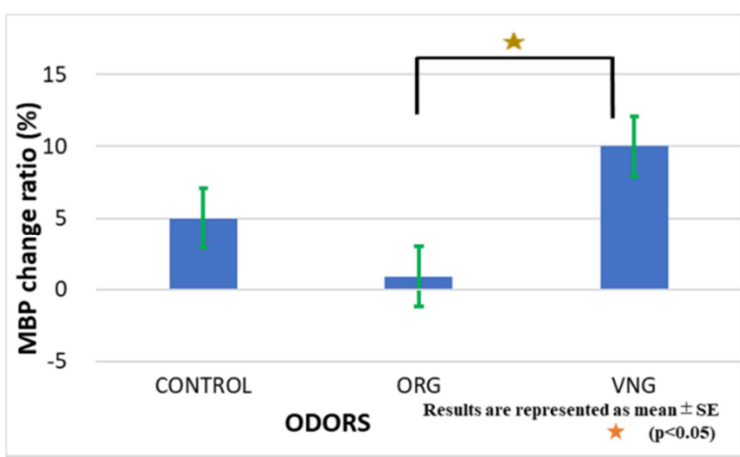

Figure 7. The effects of odors on MBP change ratio.

The change ratio $=($ post-value - pre-value $) /$ pre-value $* 100$. CONTROL: without odor inhalation, ORG: orange odor inhalation, VNG: vinegar odor inhalation. There was a main effect of odor in mean blood pressure change ratio $(\mathrm{p}<0.05)$. Turkey post-hoc revealed that mean blood pressure change ratio for VNG was significantly higher than those for ORG $(\mathrm{p}<0.05)$. 
$\mathrm{LF} / \mathrm{HF}$ component is known as an indicator of sympathetic nerve activity and HF component is known as that of parasympathetic nerve activity $[11,12]$. To date, it is assumed that VNG, where subjects had shown negative valence and high arousal, caused activation of sympathetic nerve activity and/or withdrawal of parasympathetic nerve activity. And thus, the VNG showed significant increase in LF/HF and decrease in HF components when it was compared to the control condition.

The change ratio of diastolic and mean BP for VNG was significantly higher than those for ORG (Figures 6 and 7). It is known that activation of sympathetic nerve activity causes vasoconstriction and it may consequently cause the diastolic BP increase [13]. In addition, it is said that presentation of unpleasant odor causes increase in autonomic arousal [8, 14]. Furthermore, Carlson [15] reported that both negative and positive smell evoked changes in resting state brain networks when it is compared to the no-odor condition. Thus, we assume that $\mathrm{VNG}$, where the subjects had shown negative valence and high arousal, has brought about increase in diastolic and mean BP when it was compared to ORG, where the subjects had shown low arousal and positive valence.

Contrary to our second hypothesis, neither the $\mathrm{LF} / \mathrm{HF}$ change ratio nor the HF norm change ratio revealed a significant difference between ORG and the control. These results were not consistent with previous study [16] which dealt with another citrus called fruit-yuzu. Possible reasons are as following. An arousal lowering (calming) effect of ORG might be less than we commonly believed. And/or the concentration of orange odor might be too low to affect autonomic nerve activities as threshold values are significantly influenced by concentration [17].

\section{Conclusion}

Breathing VNG suggests that odors causing negative valence and high arousal can facilitate sympathetic nerve activity, and/or withdraw para-sympathetic nerve activity. A higher BP with VNG compared to ORG can also be attributed to the effect of these autonomic nerve activities. Consider all the various factors together, it can be concluded that some emotionally linked odors were strong enough to modulate autonomic nerve activities as well as the level of BP in resting individuals. It is thought to be of interest if the future study is directed to the investigation of odor effects on muscle responses which would relate to autonomic nerve activities.

\section{Acknowledgements}

The authors express their appreciation to Mr. Doumoto for his help in this research.

The authors further extended their thanks to all the subjects who underwent the assessment to provide the data needed for the work.

Last but certain not least, thanks are given to their research assistants who helped in assessments of the subjects and kept a smooth professional flow to the data gathering process of the study.

This work is supported by JSPS KAKENHI Grant Number JP $18 \mathrm{~K} 06454$.

\section{References}

[1] Nagai K: The influence of olfactory stimulus on energy metabolism and its mechanism-The effects of grapefruit and lavender scent (in Japanese), Journal of Japan Society for the Study of Obesity, 2006, 11, 92-94.

[2] Nagai K, Niijima A, Horii Y, Shen J, and Tanida M: Olfactory stimulatory with grapefruit and lavender oils change autonomic nerve activity and physiological function, Autonomic Neuroscience, 2014, 185, 29-35.

[3] Haze S, Sasaki K and Gozu Y: Effects of fragrance on sympathetic activity in normal adult, Japan Journal of Pharmacology, 2002, 90, 247-253.

[4] Kanzaki S: Time course of autonomic nerve function affected by olfactory (aroma) stimulation (abstract in English), Annual report of cosmetology, 2015, 23, 148-154.

[5] Yoshida S, and Saeki Y: Effects of fragrance on autonomic nervous system, (abstract in English), Journal of Japanese Society of Nursing Research, 2000, 23, 11-17.

[6] Kawai F: A research on physiological effects of odorants in cosmetics on human autonomic nerve system (abstract in English), Annual report of cosmetology, 2007, 15, 120-124.

[7] Mori H, Kobayashi A, Kikkawa S, and Yamashita H: Effects of essential oil fragrances and preference thereof on blood pressure and heart rate in healthy human subjects (abstract in English), Japanese Journal of Complementary and Alternative Medicine, 2009, 6, 137-142.

[8] Anderson AK, Christoff K, Stappen I, Panits D, Ghahremari DG, and Glover G: Dissociated neural representations of intensity and valence in human olfaction, Nature Neuroscience, 2003, 6, 196-202.

[9] Winston JS, Gottfried JA, Kilner JM, and Dolan RJ: Integrated neural representations of odor intensity and affective valence in human amygdala, The Journal of Neuroscience, 2005, 25, 8903-8907.

[10] Zawadzki MJ, Mendiola J, Walle EA, Gerin W: Between-person and within-person approaches to the prediction of ambulatory blood pressure: the role of affective valence and intensity, Journal of Behavioral Medicine, 2016, 39, 757-766.

[11] Kobayashi $\mathrm{H}$, Ishibashi $\mathrm{K}$ and Noguchi $\mathrm{H}$ : Heart Rate Variability -An Index for Monitoring and Analyzing Human Autonomic Activities, Journal of Physiological Anthropology, $1999,18,53-59$.

[12] Task Force of the European Society of Cardiology the North American Society of Pacing Electrophysiology: Heart Rate Variability Standards of Measurement, Physiological Interpretation, and Clinical Use, Circulation, 1996, 93, 10431065 .

[13] Wilmore JH and Costill DL; Physiology of Sport and Exercise (2nd ed.); Human Kinetics, Champaign, 1999, 69-70. 
[14] Ismaili OA, Maury EV, Dittmar A, Delhomme G, and Chanel J: Odor hedonics - Connection with emotional response estimated by autonomic parameters, Chemical Senses, 1997, $22,237-248$.

[15] Carlson H, Leitao J, Delplanque S, Cayeux I, Sander D, and Vuilleumier P: Sustained effects of Pleasant and Unpleasant Smells on Resting State Brain Activity, Cortex, 2020, 132, 386-403.
[16] Matsumoto T, Kimura T, and Hayashi T: Aromatic effects of a Japanese citrus fruit-yuzu (Citrus junos Sieb. ex Tanaka)-on psychoemotional states and autonomic nervous system activity during the menstrual cycle: a single-blind randomized controlled crossover study, BioPsychoSocial Medicine, 2016, PMC4839105.

[17] Tonacci A, Billeci L, Mambro I D, Marangoni R, Sanmartin C, and Venturi F: Wearable Sensor for Assessing the Role of Olfactory Training on the Autonomic Response to Olfactory Stimulation, Sensors, 21, 2021, PMC7865293. 